GLASNIK MATEMATIČKI

Vol. 38(58)(2003), $19-27$

\title{
ON STABILITY OF CRITICAL POINTS OF QUADRATIC DIFFERENTIAL EQUATIONS IN NONASSOCIATIVE ALGEBRAS
}

\author{
Borut Zalar and Matej Mencinger \\ University of Maribor, Slovenia
}

\begin{abstract}
In this note we treat the stability of nonzero critical points of the differential equation $\dot{x}=x^{2}$ in a commutative real nonassociative algebra. As our first result we prove that if a critical point lies in some Peirce subspace with respect to a nonzero idempotent, it cannot be stable. This improves a previously known result due to Kinyon and Sagle. As a second result we show that there exists 2-dimensional algebra, with a nonzero critical point and a nontrivial idempotent, such that the critical point is stable, so that the additional assumption in our first result cannot be completely lifted.
\end{abstract}

\section{INTRODUCTION}

One possibility for approaching polynomial systems of autonomous differential equations in $\mathbb{R}^{n}$ is via the theory of commutative (nonassociative in general) finite dimensional algebras. It seems that this idea originated with Markus in [Mar]. A standard reference for the state of art in 1990, with many references to older papers, is [Wal1]. Some last decade papers of importance are [GW, Hop, KS1, KS2, Myu, Wal2].

For every autonomous polynomial system of ODE it is possible to construct a homogeneous quadratic system of ODE such that the original solutions can be read off the new solutions. For detail of this construction the reader should consult [Wal1, KS2]. The quadratic form in the quadratic system $\dot{x}=Q(x)$ can be interpreted as the diagonal of a bilinear form, i.e. $Q(x)=B(x, x)$. Defining $x \cdot y=B(x, y)$ we can interpret the obtained system

2000 Mathematics Subject Classification. 34A34,17A99.

Key words and phrases. Quadratic differential equation, nonassociative algebra, critical points, ray solutions, projections, nilpotents, Peirce subspaces. 
of ODE as a quadratic equation $\dot{x}=x^{2}$ in a finite dimensional algebra. As an example we note that the system

$$
\begin{aligned}
& \dot{x}=a x^{2}+2 b x y+c y^{2} \\
& \dot{y}=d x^{2}+2 e x y+f y^{2}
\end{aligned}
$$

can be viewed as a quadratic equation corresponding to the algebra whose multiplication table is given by

\begin{tabular}{c|c|c|}
$\cdot$ & $i$ & $j$ \\
\hline$i$ & $a i+d j$ & $b i+e j$ \\
\hline$j$ & bi+ej & $c i+f j$ \\
\hline
\end{tabular}

Under this interpretation critical points correspond to nilpotents of order two, i.e. $n^{2}=0$, while ray solutions correspond to idempotents, i.e. $p^{2}=p$. For applications of these and other algebraic concepts in the theory of ODE we again refer to the above mentioned references.

The topic of stability of critical points of the quadratic equation $\dot{x}=x^{2}$ was taken up in [KS1] which is also a starting point for the present investigation. Among other things Kinyon and Sagle proved

- if algebra has a nonzero idempotent, then 0 (which clearly is always a critical point) is not stable;

- the critical point 0 can never be asymptotically stable;

- if $p$ is an idempotent and a (nonzero) critical point lies in the Peirce subspace $A_{\frac{1}{2}}(p)$, then it is not stable.

The last result (Corollary 3.8 of [KS1]) is a direct motivation of the present paper and we intend to show that the spectral value $\frac{1}{2}$ can in fact be replaced by an arbitrary real $\lambda$ while the conclusion still remains true. As an interesting remark we note that it was proved a long time ago by Röhrl and by Kaplan and Yorke independently that every finite dimensional real algebra contains at least one nonzero idempotent or nilpotent (see [KY, Roh]).

\section{Critical points lying in Peirce subspaces}

Let $A$ be a finite dimensional real commutative algebra, which is not assumed to be associative. Let $p \in A$ be a nonzero idempotent and $n \in A$ a nilpotent of order 2 . As we mentioned in the introduction, $n$ is a critical point of the quadratic equation $\dot{x}=x^{2}$. If $n=0$, it was already proved by Kinyon and Sagle that $n$ is not stable, so we assume in the sequel that $n$ is also nonzero.

If $\lambda$ is a real constant, we can define

$$
A_{\lambda}(p)=\{x \in A: p \cdot x=\lambda x\}
$$

which can be viewed as an eigenspace of the multiplication operator $L_{p}$ : $x \longmapsto p \cdot x$. For almost all values of $\lambda$ we shall have $A_{\lambda}(p)=\{0\}$. If this is 
not the case, we can call $\lambda$ the eigenvalue of $p$ and $A_{\lambda}(p)$ is the Peirce $\lambda-$ subspace. The reader is perhaps familiar with the theory of Jordan algebras, where one of the crucial results states that the only possible eigenvalues are 0,1 and $\frac{1}{2}$. In general algebras this is not true as any value of $\lambda$ can occur. The only generally valid remark (trivial) is that $p \in A_{1}(p)$, so 1 is always an eigenvalue. The purpose of this section is to prove

TheOREM 2.1. If the critical point $n$ belongs to the Peirce $\lambda$ - subspace for some $\lambda$, then $n$ is not stable.

With the correct idea the proof appears to be remarkably simple.

Proof. Let $\epsilon$ be a positive constant. Every neighborhood of the critical point $n$ contains a point of the form $n+\epsilon p$ for some $\epsilon>0$. We shall prove that the solution of the equation $\dot{x}=x^{2}$ satisfying the initial condition $x(0)=$ $n+\epsilon p$, where $\epsilon$ is small enough, goes to infinity. This clearly implies that $n$ cannot be stable.

Since $n$ belongs to $\lambda$-subspace, we have $p n=\lambda n$. We shall seek the solution in the form

$$
x(t)=f(t) p+g(t) n,
$$

where $f, g$ are real functions. Since $n, p$ are nonzero, one being idempotent another nilpotent, they are linearly independent. The equation $\frac{d x}{d t}=x^{2}$ then gives

$$
\begin{aligned}
\frac{d f}{d t} p+\frac{d g}{d t} n & =(f p+g n)^{2}=f^{2} p^{2}+2 f g p n+g^{2} n^{2}= \\
& =f^{2} p+2 \lambda f g n+0,
\end{aligned}
$$

which implies

$$
\begin{aligned}
& \frac{d f}{d t}=f^{2}, \\
& \frac{d g}{d t}=2 \lambda f g,
\end{aligned}
$$

where our initial conditions are

$$
\begin{aligned}
& f(0)=\epsilon>0, \\
& g(0)=1 .
\end{aligned}
$$

It is quite easy to solve this explicitly and obtain the solution

$$
x(t)=\frac{\epsilon}{1-\epsilon t} p+\left(\frac{1}{1-\epsilon t}\right)^{2 \lambda} n
$$

which is well-defined on the interval $\left[0, \frac{1}{\epsilon}\right)$ for every real $\lambda$ and even has a finite escaping time property. 


\section{Two Dimensional COUnterexample}

After the result in the previous section one might ask a natural question whether with some additional work it may be possible to prove an even much more general result, i.e. that in the presence of idempotents (ray solutions) no critical point can be stable. This however is not true. Now we show that already among two dimensional algebras containing idempotents there are such for which the system $\dot{x}=x^{2}$ has stable critical points.

Let a commutative real algebra $A$ be given with the following multiplication table

\begin{tabular}{c|c|c|}
$\cdot$ & $\mathrm{n}$ & $\mathrm{a}$ \\
\hline $\mathrm{n}$ & 0 & $-\mathrm{a}$ \\
\hline $\mathrm{a}$ & $-\mathrm{a}$ & $-\mathrm{n}$ \\
\hline
\end{tabular}

It is obvious that $A$ contains a nilpotent $n$ of order two which is a critical point of the system $\dot{x}=x^{2}$. Further we have

Proposition 3.1. The algebra $A$ has exactly two idempotents $p=-\frac{1}{2} n+$ $\frac{1}{\sqrt{2}} a$ and $q=-\frac{1}{2} n-\frac{1}{\sqrt{2}} a$. They satisfy $p q=-\frac{1}{2}(p+q)$ and both have $1,-\frac{1}{2}$ as eigenvalues. Furthermore

$$
A=A_{1}(p) \oplus A_{-\frac{1}{2}}(p)=A_{1}(q) \oplus A_{-\frac{1}{2}}(q)
$$

where

$$
A_{1}(p)=\mathbb{R} p, \quad \mathbb{A}_{1}(q)=\mathbb{R} q, \quad \mathbb{A}_{-\frac{1}{2}}(p)=\mathbb{R}(p+3 q), \quad A_{-\frac{1}{2}}(q)=\mathbb{R}(q+3 p) .
$$

With respect to those idempotents the critical point can be decomposed as

$$
\begin{aligned}
& n=-\frac{2}{3} \cdot p-\frac{1}{3} \cdot(p+3 q), \\
& n=-\frac{2}{3} \cdot q-\frac{1}{3} \cdot(q+3 p) .
\end{aligned}
$$

Proof. The equation

$$
(\alpha n+\beta a)^{2}=\alpha n+\beta a
$$

gives, using the above mentioned table, the system for two real variables

$$
\begin{aligned}
\alpha & =-\beta^{2}, \\
-2 \alpha \beta & =\beta,
\end{aligned}
$$

whose nonzero solutions are

$$
\alpha=-\frac{1}{2}, \quad \beta=-\frac{1}{\sqrt{2}},
$$

hence $p$ and $q$ are obtained. Then it can be directly verified that $p q=-\frac{1}{2}(p+$ $q)$. The Peirce decomposition can be obtained via the equation

$$
p(\alpha p+\beta q)=\lambda \alpha p+\lambda \beta q
$$


which implies

$$
\begin{array}{r}
\alpha-\frac{\beta}{2}=\lambda \alpha, \\
-\frac{\beta}{2}=\lambda \beta
\end{array}
$$

whose nonzero solutions are possible when $\lambda=1(\beta=0)$ or $\lambda=-\frac{1}{2}(3 \alpha=\beta)$. In a similar way we find the decomposition with respect to $q$. The decomposition of $n$ is then obtained from the relation $p=-\frac{1}{2} n+\frac{1}{\sqrt{2}} a=-\frac{1}{2} n+\frac{1}{2}(p-q)$.

We have seen that our algebra can also be given with the multiplication table

\begin{tabular}{c|c|c|}
$\cdot$ & $\mathrm{p}$ & $\mathrm{q}$ \\
\hline $\mathrm{p}$ & $\mathrm{p}$ & $-\frac{1}{2}(p+q)$ \\
\hline $\mathrm{q}$ & $-\frac{1}{2}(p+q)$ & $\mathrm{q}$ \\
\hline
\end{tabular}

The equation $\dot{x}=x^{2}$, written in this basis becomes

$$
\frac{d f}{d t} \cdot p+\frac{d g}{d t} \cdot q=f^{2} \cdot p+g^{2} \cdot q-f g \cdot p-f g \cdot q
$$

and therefore we consider the following system (called BS in the sequel) of ODEs

$$
\begin{aligned}
& \frac{d f}{d t}=f^{2}-f g \\
& \frac{d g}{d t}=g^{2}-f g .
\end{aligned}
$$

All points of the form $\alpha p+\alpha q$ are obviously critical points and we shall see that for negative $\alpha$ they are stable. In order to simplify further writing we study only the critical point $n=-p-q$ so we are interested in the behavior of the solutions $(f, g)$ of $(\mathrm{BS})$ in the neighborhood of the critical point $(-1,-1)$. Note that we choose among possible inner products on $A$ the product in which $\{p, q\}$ is an orthonormal base. We shall prove that $n$ is stable by showing that for all initial conditions $x_{0}$ which are not too far from $n$ there exists a solution of (BS) which is defined for all positive times and stays within the circle with center at the critical point and passing through $x_{0}$.

LEMMA 3.2. The solutions of $(B S)$ lie on hyperbolas $f g=$ const.

Proof. Since

$$
\begin{aligned}
\frac{d(f g)}{d t} & =\frac{d f}{d t} \cdot g+f \cdot \frac{d g}{d t}= \\
& =\left(f^{2}-f g\right) g+f\left(g^{2}-f g\right)=0
\end{aligned}
$$

the result follows. 
LEMma 3.3. Every point $(-\gamma,-\gamma)$, where $\gamma<0$, is a critical point of $(B S)$ and attracts all points on the hyperbola $f g=\gamma^{2}$ which lie in the third quadrant.

Proof. Consider a point $\left(f_{0}, g_{0}\right)$ on the hyperbola $f g=\gamma^{2}$ with $f_{0}$ and $g_{0}$ being negative. We are looking for the solutions of (BS) with this point as initial condition. By Lemma 3.2 the first equation of (BS) becomes

$$
\frac{d f}{d t}=f^{2}-\gamma^{2}
$$

whose general solution is

$$
f(t)=\gamma \frac{1+\delta e^{-2 \gamma t}}{-1+\delta e^{-2 \gamma t}}
$$

In a similar way we obtain

$$
g(t)=\gamma \frac{-1+\delta e^{-2 \gamma t}}{1+\delta e^{-2 \gamma t}}
$$

Solving for initial conditions

$$
\begin{aligned}
& f_{0}=\gamma \frac{1+\delta}{-1+\delta} \\
& g_{0}=\gamma \frac{-1+\delta}{1+\delta}
\end{aligned}
$$

we obtain

$$
\begin{aligned}
& \gamma=\sqrt{f_{0} g_{0}}, \\
& \delta=\frac{\sqrt{\frac{f_{0}}{g_{0}}}-1}{\sqrt{\frac{f_{0}}{g_{0}}}+1} .
\end{aligned}
$$

Since the absolute value of $\delta$ is obviously smaller than 1 , this solution is defined for all positive times. It is also elementary to verify that

$$
x(t)=(f(t), g(t)) \longrightarrow(-\gamma,-\gamma)
$$

as $t \rightarrow \infty$.

Denote by $G=B\left(n, \frac{1}{\sqrt{2}}\right)$ the open ball with radius $\frac{1}{\sqrt{2}}$ centered at $n$. It is obvious that all points $\left(f_{0}, g_{0}\right) \in G$ have both coordinates negative. In the sequel we need some numerical facts about $G$. They are summarized below. The proof is a rather straightforward exercise in calculus, i.e. computation of extremal values on bounded subsets of $\mathbb{R}^{2}$, so we omit details. 
Lemma 3.4. Let $\left(f_{0}, g_{0}\right) \in G$. Then the following holds

$$
\begin{aligned}
\left(-\sqrt{f_{0} g_{0}},-\sqrt{f_{0} g_{0}}\right) & \in G, \\
\sqrt{-f_{0}}+\sqrt{-g_{0}} & >\sqrt{2}, \\
f_{0} g_{0} & >\frac{1}{4} .
\end{aligned}
$$

Lemma 3.5. For all initial conditions $x_{0} \in G$ the solution $x(t)=$ $(f(t), g(t))$ of $(B S)$ has the property

$$
\operatorname{dist}\left(n, x_{\infty}\right) \leq \operatorname{dist}\left(n, x_{0}\right) .
$$

ProOF. We must prove that

$$
2\left(1-\sqrt{f_{0} g_{0}}\right)^{2} \leq\left(1+f_{0}\right)^{2}+\left(1+g_{0}\right)^{2}
$$

for all $\left(f_{0}, g_{0}\right) \in G$. We can first expand the above inequality into

$$
f_{0}^{2}+g_{0}^{2}+2 f_{0}+2 g_{0}-2 f_{0} g_{0}+4 \sqrt{\left(-f_{0}\right)\left(-g_{0}\right)} \geq 0 .
$$

and then factor it as

$$
\left(-f_{0}-g_{0}+2 \sqrt{f_{0} g_{0}}-2\right)\left(\sqrt{-f_{0}}-\sqrt{-g_{0}}\right)^{2} \geq 0 .
$$

The last factor is always nonnegative, while Lemma 3.4 implies

$$
2<\left(\sqrt{-f_{0}}+\sqrt{-g_{0}}\right)^{2}=-f_{0}-g_{0}+2 \sqrt{f_{0} g_{0}}
$$

so the first factor is positive as well.

LEMma 3.6. For all initial conditions $x_{0}=\left(f_{0,} g_{0}\right) \in G$ the solution $x(t)=(f(t), g(t))$ of $(B S)$ has the property that the distance function

$$
D(t)=\operatorname{dist}(n, x(t))
$$

has no local extrema. Thus, combined with the above lemma,

$$
D(t) \leq D(0)=\operatorname{dist}\left(n, x_{0}\right)
$$

Proof. We know that the solution lies on the hyperbola $f g=\gamma^{2}$ between points $\left(f_{0}, g_{0}\right)$ and $(-\gamma,-\gamma)$. We must therefore investigate the function

$$
\begin{gathered}
D(f, g)=(1+f)^{2}+(1+g)^{2}= \\
(1+f)^{2}+\left(1+\frac{\gamma^{2}}{f}\right)^{2} .
\end{gathered}
$$

After taking derivative we obtain the following condition for extrema

$$
(1+f)-\left(1+\frac{\gamma^{2}}{f}\right) \frac{\gamma^{2}}{f^{2}}=0
$$

which can be factored as

$$
(f-\gamma)(f+\gamma)\left(f^{2}+f+\gamma^{2}\right)=0 .
$$


The first factor is always nonzero since $f$ is negative. The second one represents the limit value $x_{\infty}=(-\gamma,-\gamma)$ which cannot be attained at any finite time $t$. Further, Lemma 3.4 tells us $\gamma^{2}>\frac{1}{4}$. This clearly implies that the equation

$$
f^{2}+f+\gamma^{2}=0
$$

has no real solutions, and so the last factor is strictly positive even for all real $f$.

All these results combined show us that for every $\epsilon>0$ we have a $\delta=$ $\min \left\{\frac{1}{\sqrt{2}}, \varepsilon\right\}$ such that when the initial condition is in $\delta$ - neighborhood of the critical point $n$, then the solution stays within $\epsilon$-neighborhood for all later times. Therefore we have

THEOREM 3.7. There exists a real algebra with a nonzero idempotent and a nonzero nilpotent of order two such that the latter is a stable critical point of the quadratic system $\dot{x}=x^{2}$.

Remark 3.8. By the result of Kinyon and Sagle mentioned in the introduction we know that this system also has a nonstable (namely 0) critical point. Since $n$ lies on a line wholly consisting of critical points, the point $n$ is not asymptotically stable. In the case of general algebras it seems that the problem of stability of nilpotents (necessary and sufficient algebraic conditions) is still wide open.

\section{ACKNowledgements.}

The authors wish to thank the referee for his suggestion which considerably improved the presentation of our work.

\section{REFERENCES}

[GW] H. Gradl and S. Walcher, Bernoulli algebras, Comm. Algebra 21 (1993), 3503-3520.

[Hop] N.C. Hopkins, Quadratic differential equations in graded algebras, in: Nonassociative Algebras and Applications, 179-182, Kluwer, Dordrecht, 1994.

[KY] J.L. Kaplan and J.A. Yorke, Nonassociative real algebras and quadratic differential equations, Nonlinear Anal. 3 (1977), 49-51.

[KS1] M.K. Kinyon and A.A. Sagle, Quadratic systems, blow-up and algebras, in: Nonassociative Algebras and Applications, 367-371, Kluwer, Dordrecht, 1994.

[KS2] M.K. Kinyon and A.A. Sagle, Quadratic dynamical systems and algebras, J. Differential Equations 117 (1995), 67-126.

[Mar] L. Markus, Quadratic differential equations and nonassociative algebras, Ann. Math. Studies 45 (1960), 185-213.

[Myu] H.C. Myung, Note on Jacobi elliptic functions, J. Algebra 200 (1998), 134-140.

[Roh] H. Röhrl, A theorem on nonassociative algebras and its applications, Manuscripta Math. 21 (1977), 181-187.

[Wal1] S. Walcher, Algebras and Differential Equations, Hadronic Press, Palm Harbor, 1991.

[Wal2] S. Walcher, Algebraic structures and differential equations, in: Jordan Algebras, 319-326, de Gruyter, 1994. 
B. Zalar

Department of Basic Sciences

Faculty of Civil Engineering

University of Maribor

Smetanova 17, 2000 Maribor

Slovenia

E-mail: borut.zalar@uni-mb.si

M. Mencinger

Department of Basic Sciences

Faculty of Civil Engineering

University of Maribor

Smetanova 17, 2000 Maribor

Slovenia

E-mail: matej.mencinger@uni-mb.si

Received: 13.03.2001.

Revised: 22.02.2002. 